\title{
Ginzburg-Landau theory of the upper critical field in layered local-pair superconductors
}

\author{
Z. Gedik \\ Department of Physics, Bilkent University, Bilkent 06533, Ankara, Turkey
}

(Received 2 September 1993)

\begin{abstract}
The upper critical field for coupled layered superconductors with local pairs is analyzed within the mean-field approach for the anisotropic negative- $U$ Hubbard model. Effects of reduced dimensionality are investigated by solving the Ginzburg-Landau equations to obtain critical fields for different anisotropy ratios. The Harper's type system of equations is solved analytically for some special field values. Limitations of Peierls substitution are also discussed.
\end{abstract}

The negative- $U$ Hubbard model has been widely used to understand the physics of local-pair superconductors with nonretarded interactions. ${ }^{1}$ The ratio of the on-site attractive interaction $U$ to the band width determines the coupling regime. When the ratio is small, the usual weak coupling or BCS picture is valid. On the other hand, for strong coupling, electrons are tightly bound to form on-site pairs and the transition to the superconducting phase is due to Bose-Einstein condensation of these hard core bosons. Due to their very small coherence lengths, the high- $T_{c}$ cuprate superconductors are nearer to the latter limit. It is the aim of this study to analyze the upper critical magnetic fields for layered systems in the large $U$ or strong coupling regime.

The Ginzburg-Landau (GL) functional for layered systems has been treated by mean-field theory at both weak ${ }^{2}$ and strong ${ }^{3}$ coupling limits. Wen and $\mathrm{Kan}^{3}$ have investigated the case of the quasi-two-dimensional charged Bose gas. In this paper the same problem is solved on a lattice.

In the strong coupling limit, the negative- $U$ Hubbard model can be mapped onto a hard core Bose gas on a lattice with an effective hopping amplitude $-2 t^{2} / U$ and nearest neighbor repulsion $2 t^{2} / U$ where $t$ is the nearest neighbor tight binding hopping matrix element for electrons. ${ }^{1}$ A hard core Bose gas on a lattice is isomorphous to a spin- $\frac{1}{2}$ problem where superfluid order corresponds to an $x-y$ ferromagnetic alignment which can be solved by means of a GL-type functional. ${ }^{4}$ It should be noted that negative- $U$ Hubbard model has solutions corresponding to charge ordered states also. As long as the filling factor $\nu=N_{e} / 2 N$, where $N_{e}$ is the number of electrons and $N$ is the number of lattice sites, is low the superconducting state is favored. However, in the very dilute limit boson-boson interactions become important and mean-field theory may not give reliable results. Therefore, using the existing phase diagrams for the model, it will be assumed that $\nu \simeq 0.1-0.2$ where the superconducting phase is guaranteed for a large range of parameters.

In order to analyze the layered structures the GL functional can be generalized to include strong anisotropy effects. This is achieved by using different hopping amplitudes, $t_{\perp}$ and $t_{\|}$, for motion perpendicular and parallel to layers, respectively. In this model a layer is assumed to be a square lattice in the $x y$ plane with a lattice constant $a_{\|}$while the layers are separated by $a_{\perp}$ in the $z$ direction. At temperature $k_{B} T$, the free energy functional up to quadratic terms in on-site order parameters $\psi_{\mathbf{r}=(l, m, n)}$ reads

$$
\begin{aligned}
F=\sum_{\mathbf{r} \mathbf{r}^{\prime}} & T_{\mathbf{r} \mathbf{r}^{\prime}} \psi_{\mathbf{r}}^{*} \psi_{\mathbf{r}^{\prime}} \exp \left(i \phi_{\mathbf{r} \mathbf{r}^{\prime}}\right)+\frac{k_{B} T}{1-2 \nu} \ln \left(\frac{1-\nu}{\nu}\right) \\
& \times \sum_{\mathbf{r}}\left|\psi_{\mathbf{r}}\right|^{2} .
\end{aligned}
$$

Here, $T_{\mathbf{r r}^{\prime}}$ is the effective boson hopping amplitude

$$
\begin{aligned}
T_{\mathbf{r \mathbf { r } ^ { \prime }}}= & -\frac{2 t_{\|}^{2}}{U} \delta_{m, m^{\prime}} \delta_{n, n^{\prime}}\left(\delta_{l+1, l^{\prime}}+\delta_{l-1, l^{\prime}}\right) \\
& -\frac{2 t_{\|}^{2}}{U} \delta_{l, l^{\prime}} \delta_{n, n^{\prime}}\left(\delta_{m+1, m^{\prime}}+\delta_{m-1, m^{\prime}}\right) \\
& -\frac{2 t_{\perp}^{2}}{U} \delta_{l, l^{\prime}} \delta_{m, m^{\prime}}\left(\delta_{n+1, n^{\prime}}+\delta_{n-1, n^{\prime}}\right)
\end{aligned}
$$

and $\phi_{\mathbf{r r}^{\prime}}$ is the phase due to vector potential $\mathbf{A}=$ $H(0, x \cos \theta, x \sin \theta)$ for the magnetic field $\mathbf{H}=$ $H(0, \sin \theta, \cos \theta)$ and it is given by

$$
\begin{aligned}
\phi_{\mathbf{r \mathbf { r } ^ { \prime }}}= & \frac{2 \pi H a_{\|} l}{\phi_{0}} \delta_{l, l^{\prime}}\left[\left(\delta_{m-1, m^{\prime}}-\delta_{m+1, m^{\prime}}\right) a_{\|} \cos \hat{\theta}\right. \\
& \left.+\left(\delta_{n-1, n^{\prime}}-\delta_{n+1, n^{\prime}}\right) a_{\perp} \sin \theta\right]
\end{aligned}
$$

where $\phi_{0}=h c / 2 e$ is the flux quantum.

The first term in Eq. (1) can be derived from the negative- $U$ Hubbard model by means of degenerate perturbation theory where the effective second-order Hamiltonian is given by

$$
H_{\text {eff }}=P_{0}\left(-\sum_{\mathbf{r} \mathbf{r}^{\prime}} \sum_{\mathbf{r}^{\prime \prime} \mathbf{r}^{\prime \prime \prime}} \sum_{\sigma \sigma^{\prime}} t_{\mathbf{r} \mathbf{r}^{\prime}} \exp \left(i \phi_{\mathbf{r r}^{\prime}} / 2\right) t_{\mathbf{r}^{\prime \prime} \mathbf{r}^{\prime \prime \prime}} \exp \left(i \phi_{\mathbf{r}^{\prime \prime} \mathbf{r}^{\prime \prime \prime}} / 2\right) c_{\mathbf{r} \sigma}^{\dagger} c_{\mathbf{r}^{\prime} \sigma} c_{\mathbf{r}^{\prime \prime} \sigma^{\prime}}^{\dagger} c_{\mathbf{r}^{\prime \prime \prime} \sigma^{\prime}} / U\right) P_{0} .
$$


Here, $P_{0}$ is the projection operator that restricts the action of $H_{\text {eff }}$ to the subspace of doubly occupied sites and $t_{\mathbf{r r}}$ is the hopping amplitude for electrons described by the operators $c_{\mathbf{r} \sigma}^{\dagger}$ and $c_{\mathbf{r} \sigma}$. It should be noted that along with the pair hopping terms, $H_{\text {eff }}$ contains terms describing the repulsion of neighboring pairs via virtual ionization. However, since these terms have only a constant contribution to the free energy $F$, they are not included in Eq. (1). Finally, the last term in Eq. (1) can be obtained by expanding the entropy expression in order parameter ${ }^{4}$ which is a good assumption in the vicinity of superconducting-normal transition line as long as the fluctuation effects are not important.

Minimization of the free energy with respect to $\psi_{\mathbf{r}}^{*}$ gives the GL equations. The upper critical magnetic field for perpendicular orientation $(\theta=0)$ is calculated by finding the highest eigenvalue $\tilde{T}$ for the system of equations

$$
\psi_{l-1}+2\left(\epsilon+\cos \frac{2 \pi H a_{\|}^{2} l}{\phi_{0}}\right) \psi_{l}+\psi_{l+1}=\tilde{T} \psi_{l}
$$

for given $H$ and $\epsilon=\left(t_{\perp} / t_{\|}\right)^{2}$. The fact that for the highest eigenvalue the on-site order parameters have no $m$ or $n$ dependence is stressed by using the index $l$ only. Here $\tilde{T}$ is the dimensionless temperature defined by

$$
\tilde{T}=\frac{k_{B} T U}{2 t_{\|}^{2}} \frac{1}{1-2 \nu} \ln \frac{1-\nu}{\nu} .
$$

A similar equation is satisfied for fields parallel to the layers. This time $\psi_{l}$ 's are related by

$\psi_{l-1}+2\left(1+\epsilon \cos \frac{2 \pi H a_{\|} a_{\perp} l}{\phi_{0}}\right) \psi_{l}+\psi_{l+1}=\tilde{T} \psi_{l}$.

Both Eqs. (5) and (7) are of Harper's type ${ }^{5}$ and in general their solution can be obtained numerically. However, for some special $H$ values the problem can be handled analytically.

When $H=0$, the uniform solution $\left(\psi_{l}=\right.$ const) gives the critical temperature $\tilde{T}_{0 c}=\tilde{T}_{c}(H=0)=4+2 \epsilon$. Thus, $\tilde{T}_{0 c}$ changes linearly in $\epsilon$. For $\epsilon=1$, which corresponds to simple cubic lattice, $\tilde{T}_{0 c}=6$ while for $\epsilon=0$, two-dimensional square lattice, $\tilde{T}_{0 c}=4$. The former limit (with additional condition $a_{\perp}=a_{\|}=a$ ) is the case studied by Bulaevskii et al. ${ }^{6}$

For two more values, explicit expressions for the eigenvalues can be obtained. For perpendicular orientation (in units of $\tilde{T}_{0 c}$ )

$$
\tilde{T}_{c}\left(H=\frac{\phi_{0}}{2 a_{\|}^{2}}\right)=\tilde{T}_{c}\left(\frac{\phi_{0}}{4 a_{\|}^{2}}\right)=\frac{\sqrt{2}+\epsilon}{2+\epsilon}
$$

while for fields parallel to the layers

$\tilde{T}_{c}\left(H=\frac{\phi_{0}}{2 a_{\|} a_{\perp}}\right)=\tilde{T}_{c}\left(\frac{\phi_{0}}{4 a_{\|} a_{\perp}}\right)=\frac{1+\sqrt{1+\epsilon^{2}}}{2+\epsilon}$.

When $\epsilon=1$, the two results coincide as expected. For vanishing $\epsilon$, parallel magnetic fields have no effect on superconducting properties as long as orbital degrees of freedom are considered.

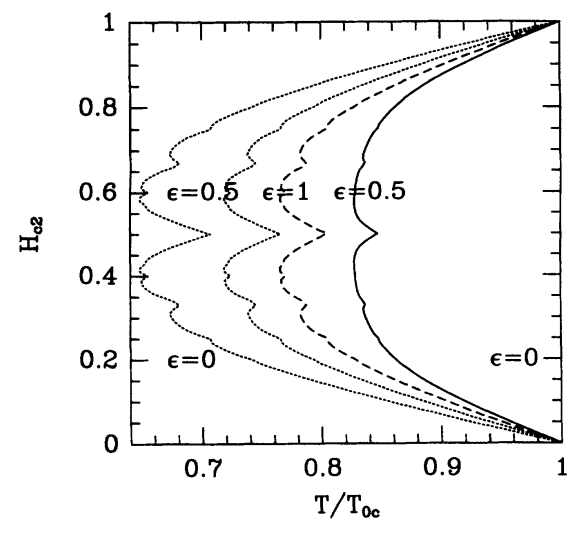

FIG. 1. Upper critical magnetic fields $H_{c 2 \perp}$ (dotted lines) and $H_{c 2 \|}$ (solid lines) vs temperature $T$ for various anisotropy ratios. $T_{0 c}$ denotes the zero field critical temperature. $H_{c 2}$ is measured in $\phi_{0} / a_{\|}^{2}$ and $\phi_{0} / a_{\|} a_{\perp}$ for perpendicular and parallel orientations, respectively. For $\epsilon=1$, which corresponds to the simple cubic lattice case, the two curves are identical (dashed line) and for the parallel fields the $\epsilon=0$ curve falls on the right axis.

Typical curves obtained by solving Eqs. (5) and (7) are shown in Fig. 1. As can be seen from the equations $T$ is periodic in $H$, if the proper units are used $\left(\phi_{0} / a_{\|}^{2}\right.$ for $H_{c 2 \perp}$ and $\phi_{0} / a_{\|} a_{\perp}$ for $\left.H_{c 2 \|}\right)$, with period unity. Furthermore, the curves are symmetric about half-integer values. In the absence of interlayer interactions, i.e., $\epsilon=0$, parallel magnetic fields have no effect on critical temperature. For this limiting value the curve falls exactly on the right axis. On the other hand $\tilde{T}_{c}$ falls to the values as low as $\sim 0.65$ for fields perpendicular to the layers. The features seen on the curves correspond to simple rational values of $H$, reflecting the fractal nature of Harper's equation. Physically, these values are attained when the flux passing through a certain number of rectangular unit cells $\left(a_{\|} \times a_{\|}\right.$or $\left.a_{\|} \times a_{\perp}\right)$ is an integer multiple of flux quantum $\phi_{0}$.

It is possible to generalize the above calculations for arbitrary orientation of the magnetic field. For $\mathbf{H}=$ $H(0, \sin \theta, \cos \theta)$, the GL equation becomes

$$
\begin{array}{r}
\psi_{l-1}+2\left(\epsilon \cos \frac{2 \pi H a_{\|} a_{\perp} \sin \theta}{\phi_{0}} l+\cos \frac{2 \pi H a_{\|}^{2} \cos \theta}{\phi_{0}} l\right) \\
\times \psi_{l}+\psi_{l+1}=\tilde{T} \psi_{l} .
\end{array}
$$

In solving Eqs. (5) and (7) it is assumed that the arguments of the cosine terms are of the form $2 \pi r l$ where $r=p / q$ is a rational number. Thus the system is periodic in $l$, and can be solved by the difference equation analog of Floquet's theorem. In order to solve the general problem by the same method, arguments of the two cosine terms in Eq. (10) should satisfy this condition simultaneously.

Although they can estimate the qualitative behavior of the physical systems correctly, mean-field calculations may not give accurate numerical results. For example, critical temperatures are overestimated in comparison to the random-phase approximation calculations. ${ }^{1}$ There- 
fore, the above calculations can be trusted only qualitatively.

Finally, it should be noted that periodic variation of $T_{c}$ with $H$ is not due to mean-field treatment of the problem but it is an artifact of Peierls substitution ${ }^{7}$ as it will be seen below. Treatment of the lattice particles of charge $q$ in magnetic fields by using the substitution

$$
T_{\mathbf{r r ^ { \prime }}} \rightarrow T_{\mathbf{r r ^ { \prime }}} \exp \left(\frac{i q}{\hbar c} \int_{\mathbf{r}^{\prime}}^{\mathbf{r}} \mathbf{A}(\mathbf{s}) \cdot d \mathbf{s}\right),
$$

where $T_{\mathbf{r} \mathbf{r}^{\prime}}$ is the hopping amplitude from site $\mathbf{r}^{\prime}$ to site $\mathbf{r}$ is due to Peierls. ${ }^{7} \mathrm{~A}$ better treatment of the problem is to take into account the fact that under magnetic field the magnitude of $T_{\mathbf{r} \mathbf{r}^{\prime}}$ changes also. Dependence of $T_{\mathbf{r r}}$ on $H$ can easily be introduced into the previous calculations. The only modification is to put the proper factor in front of the cosine terms in Eqs.(5) and (7). The dependence of the hopping amplitude on magnetic field can only be found if the atomic wave functions and pseudopotentials are known (see, for example, Ref. 8). However, the behavior of the system under strong magnetic fields is independent of the details and it is characterized by the strong suppression of $T_{\mathbf{r r}^{\prime}}$. In this limiting case cosine terms can be neglected and the solution of Eqs. (5) and (7) are given by (in units of $\tilde{T}_{0 c}$ )

$$
\lim _{H \rightarrow \infty} \tilde{T}_{c}(H)=\frac{\epsilon+1}{\epsilon+2}
$$

and

$$
\lim _{H \rightarrow \infty} \tilde{T}_{c}(H)=\frac{2}{\epsilon+2}
$$

respectively. Thus, even with the improved Peierls substitution, the mean-field theory still predicts infinite upper critical fields at low temperatures.

Divergence of the upper critical field below a certain temperature is due to decoupling of lattice sites. In this system, the vortex currents are entirely Josephson tunneling currents. At the decoupling temperature, the coherence length becomes smaller than the intersite spacing and therefore the system behaves as a collection of isolated lattice sites. Since the sites are assumed to have zero extension in space, no critical field can suppress the superconductivity.

In conclusion, the upper critical field for coupled layered superconductors with local pairs is analyzed within the mean-field approach for the anisotropic negative- $U$ Hubbard model. The $H_{c 2}(T)$ curve is found to have upward curvature near $T_{c}$, a characteristic behavior of low-dimensional superconducting systems. It is shown that periodic variation of the critical temperature with magnetic field is due to use of the Peierls substitution outside its range of validity. In this context, it is interesting to reexamine the theory of the upper critical field in layered ${ }^{9}$ and filamentary ${ }^{10}$ superconductors by using the improved Peierls substitution.
${ }^{1}$ R. Micnas, J. Ranninger, and S. Robaszkiewicz, Rev. Mod. Phys. 62, 113 (1990).

${ }^{2}$ W. E. Lawrence and S. Doniach, in Proceedings of the 12th International Conference on Low Temperature Physics, Kyoto, 1970, edited by Eizo Kanda (Academic, Tokyo, 1971), p. 361.

${ }^{3}$ Xiao-Gang Wen and Rui Kan, Phys. Rev. B 37, 595 (1988).

${ }^{4}$ L. N. Bulaevskii, A. A. Sobyanin, and D. I. Khomskii, Zh. Eksp. Teor. Fiz. 87, 1490 (1984) [Sov. Phys. JETP 60, 856 (1984)].

${ }^{5}$ P. G. Harper, Proc. R. Soc. London, Ser. A 68, 874 (1955).

${ }^{6}$ There is probably a numerical error in Fig. 1 of Ref. 4 . This can be seen by looking at special $H$ values. For example, when $H=1 / 2$ (in units of $\phi_{0} / a^{2}$ ) the problem is reduced to a one-dimensional tight binding chain with two atoms per unit cell, which can be handled analytically.

${ }^{7}$ R. E. Peierls, Z. Phys. 80, 763 (1933).

${ }^{8}$ A. Alexandrov and H. Capellmann, Z. Phys. B 83, 237 (1991).

${ }^{9}$ R. A. Klemm, A. Luther, and M. R. Beasley, Phys. Rev. B 12, 877 (1975).

${ }^{10}$ L. A. Turkevich and R. A. Klemm, Phys. Rev. B 19, 2520 (1979). 